\title{
Using High Dimensions to Compare Drawings of Graphs ${ }^{\star}$
}

\author{
Stina Bridgeman \\ Department of Mathematics and Computer Science \\ Hobart and William Smith Colleges, Geneva, NY 14456 \\ bridgeman@hws.edu
}

\section{Introduction}

We describe a simple visualization technique which allows a user to quickly assess the overall similarity of drawings of similar graphs, and to easily find regions of stability and of change. It can be used to simultaneously compare any number of drawings, and does not require that the layouts be adjusted to minimize changes.

The underlying idea - grouping vertices whose relative positions undergo little change - is shared by the animation technique of Friedrich and Houle [1, but both our approach to identifying such groups (visually via planes in high dimensions) and our application (comparing drawings) are quite different.

\section{Algorithm}

Let $D_{1}$ and $D_{2}$ be $2 \mathrm{D}$ drawings of some graph $G$, and let $\left(x_{v_{i}}, y_{v_{i}}\right)$ be the coordinates assigned to vertex $v$ in drawing $i$. Create a $4 \mathrm{D}$ drawing $D$ of $G$ in which each vertex is associated with the 4-tuple $\left(x_{v_{1}}, y_{v_{1}}, x_{v_{2}}, y_{v_{2}}\right)$, then project that $4 \mathrm{D}$ drawing into $3 \mathrm{D}$ for viewing. Groups of vertices whose relative positions are maintained under translation, rotation, and/or scaling appear on the same plane in both the $4 \mathrm{D}$ drawing $D$ and the $3 \mathrm{D}$ projection, with different planes indicating different combinations of transformations. See Figure 1.

The algorithm can be extended to include edges by evenly spacing a number of points along each edge and creating 4D "edge points" in the same manner as vertex points. Changes in the graph structure can be accommodated by assigning appropriate coordinates to the missing points. In addition, $k$ drawings can be compared simultaneously by building a $2 k$-tuple for each point from the coordinates in each drawing, then projecting to 3D for viewing.

\section{Applications}

In dynamic graph drawing and layout adjustment, the user must frequently orient herself to a new drawing of the same or nearly the same graph. The "highdimensional comparison" technique of section 2 can be used to provide visual

\footnotetext{
* Some of this work was completed while the author was a visiting researcher at the National ICT Australia (ATP Sydney) in 2007.
} 


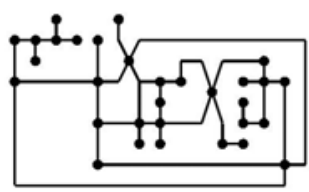

(a)

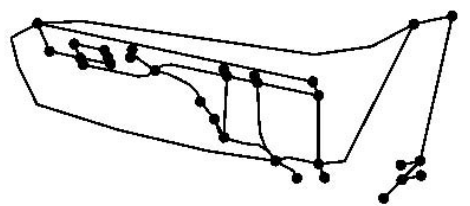

(c)

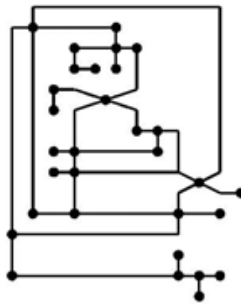

(b)

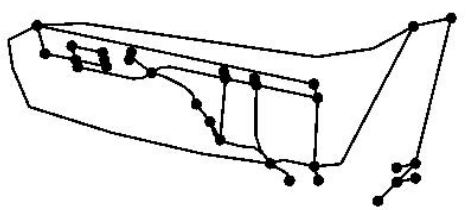

(d)

Fig. 1. $D_{1}$ and $D_{2}$ are shown in (a) and (b). Finding similar regions in these drawings requires repeatedly looking back and forth between the drawings and a lot of mental gymnastics. Figures (c) and (d) are a stereo pair showing a 3D projection of $D$ defocus your eyes until (c) and (d) merge into a third picture. (The visualization is most effective when the user can also manipulate the viewing angle to enhance the 3D sense.) Flat areas indicating similar regions can be identified immediately. Brushing can be used in an interactive viewer to match flat regions to the original drawings.

cues to aid the reorientation process, and is particularly useful when different parts of the layout have undergone different transformations (see Figure 1).

Time-varying graphs are often drawn by creating a separate layout for each time step, and either animating the transitions between time steps or stacking the layouts to create a $2.5 \mathrm{D}$ drawing. The success of these techniques requires adjusting consecutive layouts to reduce changes between time steps, which precludes the use of drawing algorithms designed to display particular structural elements of the graph (such as clusters). High-dimensional comparison provides a new way to visualize the change and stability between layers and over a series of time steps, and does not require adjustment of consecutive layouts.

In addition, the fit of the high-dimensional points to a single plane provides a potential similarity measure for comparing drawings and has the novel feature of being suitable for comparing more than two drawings at once.

Finally, several other applications are based on automatically clustering highdimensional points which lie on or near the same plane (e.g. using the techniques of Friedrich and Houle [1] or Tseng [2]). Clustering information can be used to color-code similar groups in the $D_{i}$ drawings, to produce a meta-visualization of how cluster position and membership changes over a series of drawings, or to define similarity measures based on common features rather than individual nodes. 


\section{References}

1. Friedrich, C., Houle, M.E.: Graph drawing in motion II. In: Mutzel, P., Jünger, M., Leipert, S. (eds.) GD 2001. LNCS, vol. 2265, pp. 220-231. Springer, Heidelberg (2002)

2. Tseng, P.: Nearest $q$-flat to $m$ points. J. Optim. Theory Appl. 105(1), 249-252 (2000) 